\title{
A Comparative Study between Postisometric Relaxation and Isometric Exercises in Non-Specific Neck Pain
}

Gupta*, S., Jaiswal, P. and Chhabra, D.

FIT, Department of therapies and health sciences, M. D. University, Rohtak. *Email: shilpygupta15@yahoo.co.in.

\section{Abstract}

Postisometric relaxation is commonly used to treat patients with neck pain. No randomized controlled trial examining the outcomes of this treatment in symptomatic populations has been reported in the literature. The objective of the study was to evaluate the efficacy of postisometric relaxation in patients with non-specific neck pain. A Convenient sample of thirty seven subjects diagnosed with non-specific neck pain was randomly allocated to one of the two treatment groups on the basis of the inclusion criteria. The experimental group $(n=19)$ received postisometric relaxation and control group $(n=18)$ received isometric exercises. Visual analogue scales (VAS), range of motion (ROM) and neck disability index (NDI) scores were recorded on $1^{\text {st }}$, $8^{\text {th }}, 15^{\text {th }}$ and $22^{\text {nd }}$ day. Both groups received the selected treatment for three weeks. Non-paramametric tests demonstrated a statistically significant difference with experimental group showing greater improvement in ROM, VAS and NDI than the control group and significant difference within group also. Conclusion: Postisometric relaxation may be more effective in decreasing pain and disability and increasing cervical range of motion in patients with non-specific neck pain. In order to generalize the results, the studies should be done on wider population including different subjects with different age group.

Key words: Non-specific neck pain, Postisometric relaxation, range of motion, neck disability index

\section{Introduction}

Neck pain is a common musculoskeletal disorder in the general population. Although probably not as frequent and disabling as low back pain, neck pain still constitutes a major burden on patients in terms of pain, disability and absence from work. Cote \& Cassidy (1998) reported lifetime prevalence of neck pain up to $67 \%$.

Non-specific neck pain is defined as mechanical pain located anywhere between the occiput and upper thoracic spine and surrounding muscles without any specific etiology (Gemmel and \& Miller, 2006). The International Association for the Study of Pain (IASP) has defined neck pain as: "Pain perceived as arising from anywhere within the region bounded superiorly by superior nuchal line, inferior by an unoriginally transverse line through the tip of first thoracic spinous process, and lateraly by saggital plane tangential to the lateral border of neck. A frequently seen cause of the neck pain is awkward occupational postures, heavy lifting and physically demanding work (Douglas and Bope, 2004).

Neck muscles show a strong tendency to develop hypertonus and spasm and alter proprioceptive input. Therefore; common cause of neck pain is muscle tightness. Clinically positive signs include tenderness in the posterior neck region, asymmetry, increased tension and restriction of movements (Grant, 2002). The diagnosis is mainly based on clinical examination. Various imaging techniques may be helpful in diagnosing specific conditions responsible for neck pain. Usually they are of little help when no 
underlying pathology condition is suspected.

In the review of literature, various studies revealed that specific treatment for non-specific neck pain ranges from cervical collar, cervical traction, moist heat, cervical mobilization, cervical manipulation, strengthening training routines, postural re-education, pharmacological treatment etc (Lucas et al, 2001). Manual therapy for neck pain includes manipulation and mobilization. Various studies have shown that high velocity, low amplitude techniques may correct joint restriction, but not the restriction due to muscles. Postisometric relaxation is claimed to be an effective method for acute tension in soft tissue problems that preclude immediate spinal adjustments, reduces muscle spasm that is responsible for spinal fixation, reduces pain and lengthen the tightened neck muscles to normalize gross cervical range of motion (Digiovanna \& Schiowitz, 1996) and is very effective for muscles in acute and sub-acute non-specific neck pain. This treatment is based on the mechanism of neuromuscular inhibition (Hertling and Kessler, 1996; Chaitow, 2001; Donatelli and Wooden, 2001 and Siegfried and Simons, 2001).

The present study aimed to determine the effectiveness of postisometric relaxation over the isometric exercises in non-specific neck pain, yet there is dearth of clinical trials related to the effectiveness of postisometric relaxation (Cassidy and Lopes, 1992; Bentley, 1996; Schnek and MacDiarmid, 1997; Fryer, 2000; Ballantyne \& Fryer, 2003; Lenehan \& Fryer, 2003; Wilson \& Payton, 2003; Fryer and Ruszkowski, 2004; Denise et al,
2006). No randomized controlled trial examining the outcomes of this treatment in symptomatic populations has been reported in the literature. Therefore, the purpose of the study was to compare postisometric relaxation with isometric exercises with respect to neck pain, range of motion and functional outcome in nonspecific neck pain. The research hypothesis investigated was that postisometric relaxation would be more effective than isometric exercises for patients with non-specific neck pain.

\section{Material and Methods}

This study utilized randomized controlled trial design. A convenient sample of 37 subjects in the age group of 18 to 45 years $(24.03 \pm 3.44)$ enrolled from Physiotherapy Department at Sarvodya hospital, Arya Samaj Charitable Clinic and Faridabad Institute of Health Sciences, Faridabad were included in the study after obtaining their informed consent. Out of 37 subjects, 19 subjects participated in the Experimental Group and 18 subjects in the Isometric Group. All the patient data was collected before randomization. Eligible patients were randomized to one of the two treatment groups on the basis of permuted block randomization.

Inclusion criteria included age between 18-40 years, both male and female and patients having acute or subacute non-specific neck pain. The criterion given by Lucas et al (2001) was used for defining acute or sub-acute non specific pain. As per this criterion for acute neck pain, pain should not last longer than 4 weeks and for sub acute not longer than 4-12 weeks. Subjects who had cervicogenic headache, radiculopathies, 
patients having radiating pain, severe osteopenia, whiplash associated disorders, previous cervical spine surgeries, vascular diseases of neck, progressive neurological deficit, serious medical conditions like hypertension, renal failure, rheumatoid arthritis and verteberobasilar insufficiency (VBI) were excluded from the study. These states were assessed mainly by medical history and clinical examination before the study.

Couch, Hot packs, Standard Inclinometer, Visual Analogue Scale (VAS) and Neck disability index (NDI) were the instruments used during the study ((Youdas \& Garret, 1992; Cynthia, 1998; Bronfort, et al, 2001; Werner, 2001)

The study protocol was reviewed and approved by the Institutional Research Committee of Department of Therapies and Health Sciences, Faridabad. After the process of screening, examination and informed consent, the patient rated their pain intensity on Visual Analogue Scale, functional outcomes on Neck Disability Index and followed by an inclinometer examination of cervical Range of Motion. Thereafter, subjects were randomly allocated to experimental group and control group. Treatment was given alternatively for four days a week and was followed for 3 weeks. Each session lasted for 30 minutes. All the subjects were taught home exercise program and postural re-education.

The subjects in the experimental group received post-isometric relaxation (PIR). In this procedure the physiotherapist and patient's force matched. Initial effort involved approximately $20 \%$ of the patient's maximum strength. Duration of contraction was 7-10 seconds. 3-5 repetitions were given and neck was gently guided to new restriction barrier (Chaitow, 2001). Subjects in the control group were treated with isometric exercises of the neck. Single series of 15 repetitions of isometrics was done in the forward, obliquely toward right and left and directly backward. Each repetition was held for 10 seconds. Post-treatment measurements of Visual Analogue Scale, Range of Motion and Neck Disability were taken for both the groups on $8^{\text {th }}, 15^{\text {th }}$ and $22^{\text {nd }}$ day. Both the groups were given home program, comprising of postural correction exercises, cervical exercisescervical range of motion exercises, scapular retraction exercises.

Subjects were advised to continue the above-mentioned exercises regularly, three times a day for three weeks.

Pain score and Range of Motion (ROM) values were recorded at the baseline, and then on $8^{\text {th }}, 15^{\text {th }}$ and $22^{\text {nd }}$ day and functional outcome were collected at the baseline and after intervention on $22^{\text {nd }}$ day.

\section{Results \& Discussion}

Due to the nature of outcome measures, non-parametric statistical analysis was used for the baseline characteristics of the two interventions. Wilcoxon test was used for within group analysis and Mann-Whitney test was used for between group analyses. The significance level set for this study was $\mathrm{p}$ $<0.05$. The software program used for the data analysis was SPSS version 12.00. Mean scores of Visual Analogue Score, Neck Disability Index and Range of Motion for cervical flexion, cervical extension, left and right lateral cervical flexion alongwith the statistical constants are enlisted in the table. 
Table: Mean values of VAS, NDI and ROM in the experimental and control groups

\begin{tabular}{|c|c|c|c|c|c|c|c|c|c|c|}
\hline \multirow[t]{3}{*}{ Variable } & \multicolumn{4}{|c|}{$1^{\text {st }}$ Day } & \multicolumn{4}{|c|}{$22^{\text {nd }}$ Day } & \multicolumn{2}{|c|}{$\begin{array}{c}\text { \% Change From } \\
\text { Day } 1 \text { TO } 22\end{array}$} \\
\hline & \multicolumn{2}{|c|}{$\begin{array}{c}\text { Experimental } \\
\text { group }\end{array}$} & \multicolumn{2}{|c|}{$\begin{array}{l}\text { Control } \\
\text { group }\end{array}$} & \multicolumn{2}{|c|}{$\begin{array}{c}\text { Experimental } \\
\text { group }\end{array}$} & \multicolumn{2}{|c|}{$\begin{array}{l}\text { Control } \\
\text { group }\end{array}$} & \multirow[t]{2}{*}{$\begin{array}{c}\text { Experimental } \\
\text { group }\end{array}$} & \multirow[t]{2}{*}{$\begin{array}{c}\text { Control } \\
\text { group }\end{array}$} \\
\hline & Mean & $\pm \mathrm{SD}$ & Mean & $\pm \mathrm{SD}$ & Mean & $\pm \mathrm{SD}$ & Mean & $\pm \mathrm{SD}$ & & \\
\hline NDI & 16.32 & 4.58 & 14.78 & 4.22 & 0.47 & 0.96 & 2.28 & 1.57 & 97.12 & 84.66 \\
\hline Cervical Flexion & 50.53 & 5.50 & 49.33 & 6.31 & 74.53 & 5.10 & 63.89 & 6.04 & 49.05 & 31.24 \\
\hline Cervical Extension & 48.53 & 7.04 & 47.62 & 5.06 & 66.74 & 3.74 & 58.83 & 4.96 & 39.43 & 24.28 \\
\hline Left Lateral Flexion & 26.68 & 3.80 & 27.50 & 4.68 & 41.40 & 2.70 & 36.94 & 4.90 & 54.65 & 35.95 \\
\hline
\end{tabular}

It is evident from the mean scores that both the postisometric relaxation and isometric exercises caused improvements in VAS, NDI and ROM with the course of treatments. Within group analysis revealed that both experimental and control groups demonstrated significant improvements $(\mathrm{P}<0.001)$ in VAS, NDI and ROM parameters. Between groups analysis revealed that percentage change in VAS score was more in the experimental group at on $22^{\text {nd }}$ day. In the experimental group during the study, pain levels as judged from VAS scores declined to 'zero' at Day $15^{\text {th }}$ with the results maintained at the total follow-up of 22 days. However, in the control group, the pain values though declined on Day 22 but without reaching zero level.

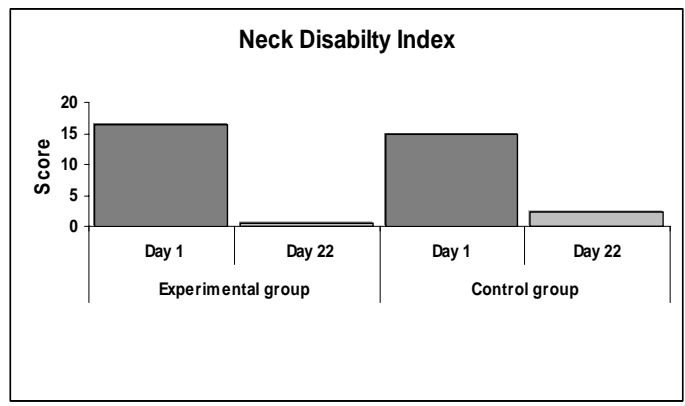

Figure 1: Changes in NDI scores in the Experimental and Control groups

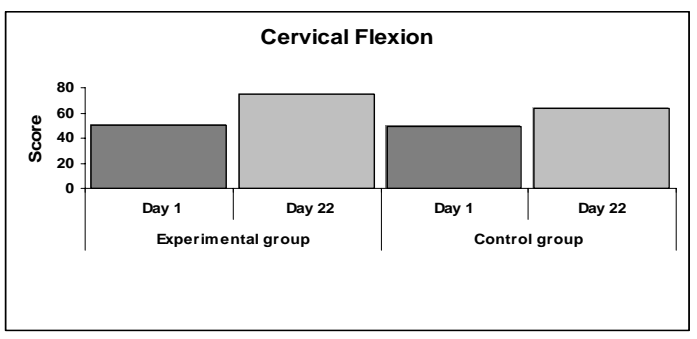

Figure 2: Changes in Cervical Flexion scores in the Experimental and Control groups

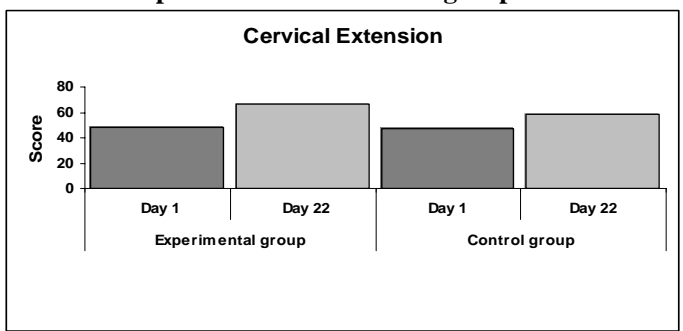

Figure 3: Changes in Cervical Extension scores in the Experimental and Control groups

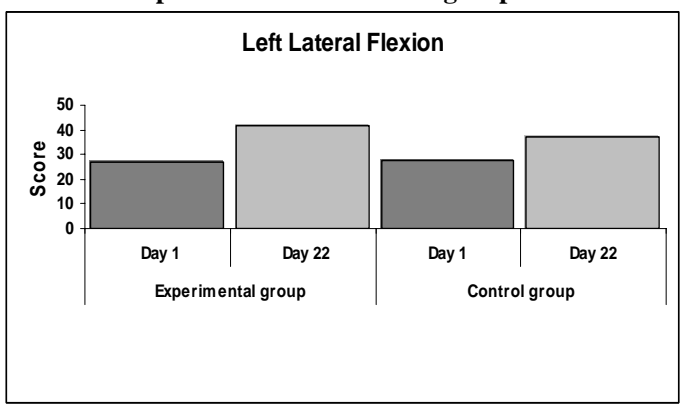

Figure 4: Changes in the Left Lateral Cervical Flexion scores in the Experimental and Control groups 


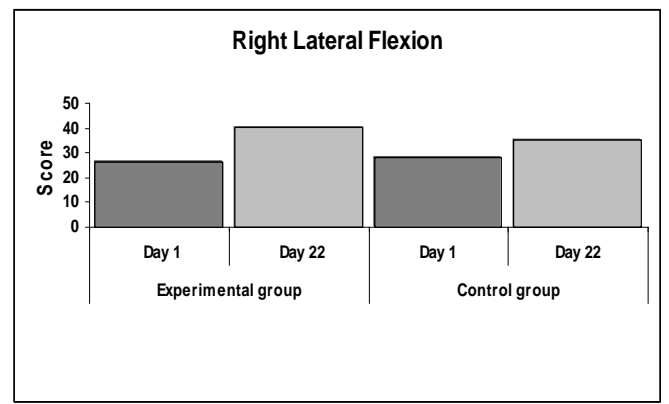

Figure 5: Changes in Right Lateral Cervical Flexion scores in the Experimental and Control groups

This study demonstrated that there was significant improvement in neck pain, range of motion and functional outcome in the experimental group as compared to the control group. Although there are numerous studies that have addressed the issue of manual therapy in neck pain, but no studies have utilized postisometric relaxation as intervention study in neck pain and so it compliments with previous studies that investigated the effect of muscle energy technique on cervical, thoracic and lumbar range of motion (Schnek \& Kimberly, 1994; Schnek, and MacDiarmid, 1997; Lenehan \& Fryer, 2003; Denise et al, 2006).

The percentage increase in range of motion was more in the experimental group as compared to the isometric group. These findings are comparable to the research of Capt. Eric Wilson, which showed that muscle energy technique decreases disability and improves function, range of motion in patients with low back pain (Wilson, \& Payton, 2003). Cassidy \& Lopes (1992) tested the immediate effect of muscle energy technique but our study tested the effect of postisometric relaxation after three weeks. So, it was difficult to compare the results of our study with this study.

Recent study clearly demonstrated significant improvement in pain (VAS), disability (NDI) and cervical range of motion in both groups (Figs 1-5). This improvement in experimental group possibly may be due to rapid hypoalgesic effects of mobilization -induced analgesia and is generally consistent with the proposed mechanisms of action for the postisometric relaxation and is used to treat somatic dysfunctions that result in cervical motion restriction.

In a recent randomized clinical trial (Bronfort et al, 2001) substantial improvement in the neck disability index was observed in the groups, but no significant differences between groups reported $(p>0.05)$. In contrast to these findings, our study demonstrated that experimental group had significantly improved in neck disability score $(\mathrm{p}<$ 001) than isometric group after three weeks of treatment. Because the neck disability index assesses different aspects of neck pain and consists of pain intensity, daily activities, it is suggested that improvement in the score might be due to combined effects of reduction in pain and improvement in neck muscle strength.

All the subjects showed marked reduction in VAS when compared to their baseline values. The improvement was more in the experimental group as compared to the control group as most of the subjects reported pain to be zero on day 15 and during subsequent follow up sessions. In the control group though there was improvement, but most of the subjects showed slight VAS sore on Day 22. Improvement might be due to improvement in cognitive perception of pain and fear avoidance belief about physical activities that may increase pain threshold and subjects who showed some VAS score at the end might be due to 
poor patient compliance towards home exercise program or because of certain abnormal work posture, but no definite reason could be stated for this trend in pain scores. It is also noted that number of treatment sessions required for the experimental group were less when compared to the control group. Most of the subjects of the experimental group showed improvement on Day15.

Range of motion improved more markedly for the experimental group as compared to the control group. The rationale behind the superiority of PIR over isometrics is reduced reflex activity associated with its technique. Postisometric relaxation modifies stretch perception as compared to isometrics and nociceptive nerve endings in the joint and muscle play important role via neurotransmitter modulation or gate control. Repetitive light muscle contractions increase venous, lymphatic drainage and relieve paraspinal congestion (Schnek, and MacDiarmid, 1997). According to J.B. Feland, maximal voluntary contractions are intense enough to produce symptoms of delayed onset muscle soreness, may increase the risk of injury and rapid fatigue from static holding caused by compression of capillaries during the contraction of muscle, which prevents sufficient blood supply of $\mathrm{O}_{2}$ and removal of waste products (Werner, 2001 and Denise et al, 2006). The current study followed the recommendations set out by the previous cervical, thoracic and lumbar MET studies as postisometric relaxation can be used in symptomatic patients.

This study supports the validity of using postisometric relaxation within cervical spine to improve ROM, pain and functions. Further studies can be done with wider sample including different subjects with different age group. Postisometric relaxation can be applied in the treatment of somatic dysfunctions not only in the spine, but also in ribs, extremities and pelvis. The results of this study are in agreement with the findings that postisometric relaxation reduces pain, disability and increases ROM and more specially, with studies illustrating the benefits in subjects with non-specific neck pain.

\section{Conclusion}

Postisometric relaxation is more effective in decreasing pain and disability and improving cervical range of motion as compared to isometric exercises over a period of three weeks in patients having non-specific neck pain.

\section{References}

Ballantyne, F., Fryer, G. 2003. The effect of muscle energy technique on hamstring flexibility: The mechanism of altered flexibility. J. A. O. A., 6(2): 59-63.

Bentley, S. 1996. Exercise induced muscle cramp propose mechanism and management. Sport Med., 21(6): 409-420.

Bronfort, G. D., Evans, R., Nelson, B. 2001. A randomized clinical trail of exercise and spinal manipulation for patients with chronic neck pain. Spine, 26(7): 788-796

Cassidy, J.D., Lopes, A. A. 1992. The immediate effect of manipulation versus mobilization on pain and range of motion in the cervical spine. A randomized controlled trial. J. Manipul. Physio. Ther., 15(9): 570-575.

Chaitow, Leon. 2001. Muscle energy technique. $2^{\text {nd }}$ ed. Churchill Livingstone, Philadelphia.

Cote, P., Cassidy, J.D. 1998. The prevalence of neck pain and related disability is the Saskatchewan population. Spine. 23: 1689-1698.

Cynthia, C. N. 1998. Measurement of joint motion. A guide to goniometery. $2^{\text {nd }}$ Ed.: Jaypee Publishers, New Delhi

Denise, K., Burns, M. and Wells, R. 2006. Gross range of motion in the cervical spine. The effects of osteopathic muscle energy technique in asymptomatic subjects. J. Osteopathic Med., 106(3): $137-142$ 
Digiovanna, E. L., Schiowitz, S. 1996. An osteopathic approach to diagnosis and treatment. $2^{\text {nd }} \mathrm{ed}$. Lippincott Raven.

Donal, D. P., McGrath, P. A. 1983. The Validation of visual analogue scales as ratio scale measures for chronic and experimental pain. Pain, 17: 45-56.

Donatelli, R. A., Wooden, M. J. 2001. Orthopaedic physical treatment. $3^{\text {rd }}$ ed.. Churchill Livingstone, Philadelphia.

Douglas, A. B. and Bope, E. T. 2004. Evaluation and treatment of posterior neck pain in family practice. Am. Board Fam. Pract., 17: S13-22.

Fryer, G. 2000. Muscle energy concepts-A need for change. J. Osteopathic Medicine, 3(2): 54-59.

Fryer, G. and Ruszkowski, W. 2004. The influence of contraction duration in muscle energy technique applied to the atlantoaxial joint. $J$. Osteopathic Med., 7(2): 79-84.

Gemmel, H. and Miller, P. 2006. Comparative effectively of manipulation, mobilization and activator instrument in treatment of non-specific neck pain: A systematic review. Chropractic and Osteopathy, 14: 7.

Grant, Ruth. Physical therapy of the cervical and thoracic spine. $3^{\text {rd }}$ ed. Churchill Livingstone, Philadelphia.

Hertling, D. and Kessler, R. M. 1996. Management of common musculoskeletal disorder Physical therapy principles and methods. $3^{\text {rd }}$ ed. Lippiincott-Raven.
Lenehan, K.L., Fryer, G. 2003. The effect of muscle energy technique on gross trunk range of motion. Journal Osteopathic Med., 6(1): 1318.

Lucas, J., Anita, H., Gross, R., Gasner, D. 2001. A critical approach of review of article articles on the effectiveness of conservative treatment for neck pain. Spine, 26:196-205

Magee, D. J. 1997. Orthopaedic physical assessment. $3^{\text {rd }}$ ed. W.B. Saunders, Philadelphia.

Schnek, R. J. and MacDiarmid, A. 1997. The effects of muscle energy technique on lumbar range of motion. J. Manipul. Physio. Ther., 5(4): 179183.

Schnek, R., Kimberly, A. 1994. The effects of muscle energy technique on the cervical range of motion. J. Man. Manipul. Ther., 40(2): 149 155.

Siegfried, M. and Simons, D. G. 2001. Muscle pain: Its Nature, diagnosis and treatment, Williams \& Wilkins, Lippincott.

Werner, K. 2001. Physical therapy for sports. $2^{\text {nd }}$ ed. W.B. Saunders.

Wilson, E., Payton, O. 2003. Muscle energy technique in patients with acute low back pain. A pilot clinical trial. J. Orthop. Phys. Ther., 33: 502512.

Youdas, J. W., Garret, T. R. 1992. Normal range of motion of the cervical spine: An initial goniometric study. Phys. Ther., 72: 770-780. 